\title{
La responsabilidad social: análisis del enfoque de ISO 26000
}

\section{RESUMEN}

El desarrollo de las actividades empresariales desde una perspectiva integral conlleva un conjunto de impactos en distintos actores, uno de ellos tiene carácter social, los que deben ser incluidos en la gestión de las organizaciones; es así, que se ha desarrollado una Norma Internacional que provee una guía para la responsabilidad social (RS) denominada ISO 26000.

El siguiente artículo tiene como objetivo análizar el enfoque conceptual de la responsabilidad social presentada por ISO y proponer un cambio de enfoque que permita definir de una manera adecuada las responsabilidades de las empresas.

Palabras clave: impactos, ISO 26000, licencia social, responsabilidad corporativa, responsabilidad social

SOCIAL RESPONSIBILITY : ANALYSIS OF THE APPROACH OF ISO 26000

\section{ABSTRACT}

The development of business activities from a holistic perspective involves a set of social impacts, which must be included in the management of organizations, so, that has developed an International Standard providing guidelines for social responsibility (RS) called ISO 26000.

The following article has as objective analyze the conceptual approach to social responsibility by ISO and to propose a change of approach to define in an appropriate manner the responsibilities of business..

Keywords: corporate responsibility, impacts, ISO 26000 , social responsibility, social license

\section{INTRODUCCIÓN}

La mayor competitividad de las empresas, el cambio del entorno en que desarrollan sus actividades y el mayor interés de relacionarse con actores cercanos a ellas que muchas veces determinar la sostenibilidad del servicio, ha generado que los enfoques de gestión relativos a la responsabilidad empresarial se renueven.

Es decir, la sostenibilidad empresarial también se sustenta en su responsabilidad con los grupos de interés (stakeholders) con los que interactúa, por ello se hace necesario incluirlos dentro de su gestión. Es así, que la Organización Internacional para la Normalización, ISO, ha desarrollado una Norma Internacional que provea una guía para la responsabilidad social (RS).

En los últimos años en el país se han desarrollado un conjunto de conflictos sociales relacionados con empresas que demuestran su importancia e incentivan su estudio.

\section{SISTEMA DE GESTIÓN DE LA EMPRESA RESPONSABLE}

Es común en la actualidad utilizar los términos de "responsabilidad social empresarial", "responsabilidad social corporativa" o "responsabilidad empresarial", ellos tienen el mismo significado, pero para no limitar sus implicaciones, en algunos países, se suele evitar el adjetivo "social". Por lo tanto, se convierte en término más amplio como "responsabilidad corporativa".

La Comisión de la Unión Europea en el año 2001 presentó el Libro Verde, en cuyo contenido define la responsabilidad corporativa como "la integración voluntaria, por parte de las empresas, de las preocupaciones sociales y medioambientales en sus operaciones comerciales y sus relaciones con sus interlocutores".

En cuanto a los sistemas de gestión de empresas responsables "hasta la fecha no existe un estándar consensuado y aceptado a nivel internacional que, además de constituirse en una referencia de obligada consulta, englobe todos los aspectos relacionados con la sostenibilidad" (Olcese y otros, 2008, p. 188).

Las principales normas relacionadas a responsabilidad empresarial son los siguientes:

* Magíster en Gestión Económica y Empresarial, ingeniero industrial, UNMSM. Profesor en la Facultad de Ingeniería Industrial, Departamento Académico de Producción y Gestión Industrial de la UNMSM. E-mail: cieandia@hotmail.com 
- SA8000: establecen directrices y exigencias en la gestión de las relaciones laborales con los empleados y con el personal de los contratistas o proveedores que colabore con la producción de bienes o servicios de las empresas.

- G3: Global Reporting Initiative, tiene como objetivo el aportar requerimientos sobre los contenidos de las memorias de responsabilidad empresarial.

- AA1000: La norma fue realizada por el ISEA(The Ínstitute of Social and Ethical Accountability) y fue concebida para lograr el compromiso activo de todos los copartícipes e interlocutores de una organización. Se trata de establecer un proceso sistemático de participación de los grupos de interés que va a generar los indicadores, metas y mecanismos de presentación de informes necesarios para asegurar su eficacia.

- Modelo EFQM de RC: EI EFQM framework for corporate social responsability ha sido diseñado en línea con el modelo EFQM (European Foundation for Quality Management) y recoge recomendaciones sobre cómo identificar, mejorar e integrar los impactos económicos, sociales y ambientales de las operaciones dentro de la política, de la estrategia y de la gestión del día a día de la organización, teniendo en cuenta a los grupos de interés de la misma.

- EFR1000: Modelo de gestión de la empresa familiarmente responsable, se ocupa de avanzar y dar respuestas en materia de responsabilidad en cuanto a la conciliación de la vida familiar y laboral, el apoyo en la igualdad de oportunidades y la inclusión de los más desfavorecidos.

- ISO 26000: pretende que las organizaciones asuman la responsabilidad por el impacto de sus actividades en la sociedad y el medio ambiente.

- Global Compact: fue promovido por la Organización de las Naciones Unidas; es un instrumento de libre adscripción por parte de las empresas, organizaciones laborales y civiles, que descansa en el compromiso de asumir y cumplir los principios del pacto.

Las normas anteriores se clasifican en tres categorías de estándares:

1. Normativas generales, los que cubren varios campos de la gestión dela responsabilidad corporativa.

2. Normativas que aportan recomendaciones para la gestión ética de las empresas.
3. Estándares que aportan directrices para el diseño y contenido de las memorias de responsabilidad corporativa.

Figura 1. Categorías para gestionar la RC

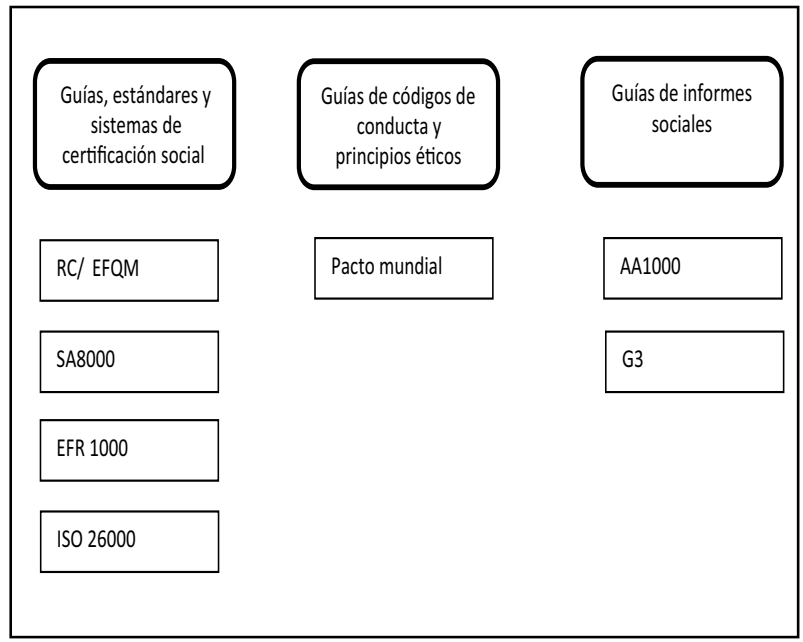

\section{RESPONSABILIDAD SOCIAL-ISO 26000}

En abril de 2001, el comité ISO sobre política de los consumidores (COPOLCO) publicó un informe sobre el valor de los estándares de responsabilidad social corporativa (RSC). En él se concluyó que, desde una perspectiva del consumidor, la ISO estaba capacitada para emprender un estándar internacional de responsabilidad social. COPOLCO sugirió entonces la necesidad de que la ISO configurara un grupo consultivo que involucrara a las principales partes interesadas con el objeto de investigar el tema en profundidad.

A partir de ahí que se creó el grupo consultivo de la ISO (SAG) para asesorar al Comité Técnico de Gerencia de la ISO (TMB) sobre responsabilidad social. En junio de 2004 se celebró la conferencia internacional de la ISO sobre responsabilidad social, organizada en Estocolmo por el Instituto Sueco de Normalización (SIS), que contó con 355 participantes de 66 y múltiples sectores. El objeto de la conferencia era debatir sobre si la ISO debía asumir el trabajo sobre responsabilidad social de las organizaciones y, de ser así, de qué manera debía hacerse.

Fruto del informe del SAG y de los alentadores resultados de Estocolmo, el consejo de gestión técnica de la ISO (TMB) propuso la creación de un nuevo grupo de trabajo (ISO/TMB/WG/SR) para elaborar un documento definitivo que brindase las directrices voluntarias sobre responsabilidad social. Así, se propuso la elaboración de un 
estándar no certificable sobre la implementación de prácticas de RS y en cuanto al cumplimiento de las organizaciones de las directrices del documento. El TMB asignó el liderazgo del grupo ISO/TMB/WG/ $\mathrm{SR}$ a los institutos nacionales de normalización de Brasil (ABNT) y Suecia (SIS). Esta idea de hermanar las representaciones de un país desarrollado con la de un país en desarrollo pretendía fortalecer las sinergias entre regiones, así como otorgar un mayor protagonismo a los países en desarrollo que, por aquel entonces, suponían 110 de los 156 países miembros de ISO.

Para dar forma al documento definitivo, se celebraron conferencias internacionales sucesivas: Salvador de Bahía y Bangkok (2005), Lisboa (2006), Sídney y Viena (2007), Santiago de Chile (2008), Quebec (2009) y Copenhague (2010). (Argandoña y Isea, 2011, 8-11) "Para el año 2010 este grupo de expertos creció en número a de 450 expertos y 210 observadores de 99 países, y 42 organizaciones internacionales y regionales" (Guía ISO 26000 Responsabilidad Social, 18 de julio 2014,1). El 1 de noviembre de este mismo año, se publicó la norma ISO 26000 de responsabilidad social a nivel mundial.

La Organización Internacional para la Normalización, ISO, ha desarrollado una Norma Internacional, una guía para la responsabilidad social (RS) por ser una necesidad de las organizaciones, tanto del sector público como de privado.

Bajo el contexto anterior se define: "ISO 26000 es una norma internacional que ofrece una guía para integrar la responsabilidad social en todo tipo de organizaciones, con el fin de ayudar a las organizaciones a contribuir al desarrollo sostenible" (Argandoña y Isea, 2011, 8-11)

La responsabilidad social está definida bajo esta norma como el compromiso de una organización ante los impactos que sus decisiones y actividades ocasionen en la sociedad y el medio ambiente mediante un comportamiento ético y transparente que contribuya al desarrollo sostenible incluyendo salud y bienestar de la sociedad. La guía y su aplicación serán voluntarias. No será una norma certificable.

De acuerdo a la ISO, la norma debería:

- Asistir a las organizaciones en la orientación de sus políticas de RS en lo referido a las diferencias en materia cultural, ambiental, y legal, además de las condiciones económicas de desarrollo.

- Proveer de una guía práctica relacionada a la operacionalización de RS, identificar y comprometerse con los stakeholders, e incrementar la credibilidad de los reportes y declaraciones hechas sobre RS.

- Poner el énfasis en el rendimiento y mejora de los resultados

- Incrementar la confianza y satisfacción de las organizaciones entre sus clientes y otros stakeholders.

- Ser consistente y no actuar en desacuerdo con los documentos ya existentes, convenios internacionales y estándares ya existentes.

- Promover la terminología común en el campo de $\mathrm{RS}, \mathrm{y}$ ampliar la conciencia en estas materias.

\section{ISO 26000 Y LA CERTIFICACIÓN}

La razón más importante de esta decisión es ser consecuente con el objetivo mismo de la norma ISO 26000: serunaguíaderesponsabilidad social paratoda clase de organizaciones. La norma está concebida para orientar a empresas, entidades del Estado, universidades, organizaciones no gubernamentales, trabajadores y hasta consumidores a un desempeño socialmente responsable, sin importar tamaño, giro, procedencia, interés o desinterés de lucro. Hacer la norma certificable concentraría la aplicación de la norma a las empresas.

En ese sentido, la ISO sostiene que la norma 26000 está pensada para millones de organizaciones y no para miles de empresas. Al estar en juego la calidad de vida de miles de millones de seres humanos y la sostenibilidad ambiental del planeta los esfuerzos deben ser de todos. No pueden existir sectores que se autoexcluyan de este compromiso. Sin certificaciones que planteen obstáculos complejos, no hay excusa para no ser parte del esfuerzo. $Y$ cada quien en su debida proporción.

\section{ENFOQUE CONCEPTUAL DE LA RESPONSA- BILIDAD SOCIAL}

La responsabilidad social es una forma ética de gestión, que implica la inclusión de las expectativas de todos los grupos de interés alrededor de la empresa por tanto, debe formar parte de la filosofía de gestión de una empresa que actúa "bien" es decir: con responsabilidad, transparencia y ética en la sociedad, por ello no debe ser vista como un conjunto de actividades de carácter social en ciertas temporadas del año ni obligatorias según normas de Estado.

Como filosofía de gestión puede ser aplicado a todo tipo de empresas e instituciones; por tal, 
es necesario incorporarlo en la planificación y desarrollo de las actividades empresariales para medirlo y evaluarlo. Además debe ser aceptada por toda la organización y ser una conducta de "todos" los integrantes de la empresa, con ello se logrará la sostenibilidad del servicio.

Los grupos de interés o stakeholders son los grupos de personas $u$ organizaciones públicos y privados a los que la empresa impacta con su actuación y quienes, por su parte, impactan en la empresa con sus conductas.

Los grupos de interés establecidos según la norma ISO 26000 , se presenta en la Figura 2, en cuya grafica se encuentra el símbolo "e", cuyo significado se refiere a la empresa.

Los colaboradores se refieren al personal de la empresa y/o organización; el gobierno representa a las organizaciones gubernamentales que regulan el funcionamiento de las empresas (impuestos, normas ambientales, etc.). La comunidad son las personas y/o organizaciones cercanas relacionadas en forma directa o indirectamente a las actividades de la empresa; el medio ambiente se refiere al entorno directo donde opera o distribuye sus productos o presta servicios la empresa.

\section{ANÁLISIS DE LOS GRUPOS DE INTERÉS PROPUESTOS POR ISO 26000}

Todas las organizaciones en el desarrollo de sus actividades generan un conjunto de impactos o cambios a sus grupos de interés. Dichos grupos se pueden clasificar en: internos (colaboradores y accionistas) así como externos (cliente, proveedores, comunidad, gobierno).

Las empresas para que sean sostenibles deben actuar bajo las tres dimensiones: económica (generar la suficiente rentabilidad económica que compense a sus accionistas, colaboradores, proveedores y al Estado), ambiental (lograr una certificación ambiental que demuestre que no afecta el medio ambiente) y social (actuar responsablemente en sus grupos de interés) según el esquema que se presenta en la Figura 3, resume el enfoque planteado.

La dimensión económica incluye los intereses de los accionistas, expresada en una rentabilidad mínima deseada; además, se incluyen los intereses de los proveedores, colaboradores, al gobierno y a los clientes.

Es decir, al lograr una rentabilidad adecuada se satisface la expectativa del accionista pero solo

Figura 2. Modelo de RS de ISO 26000

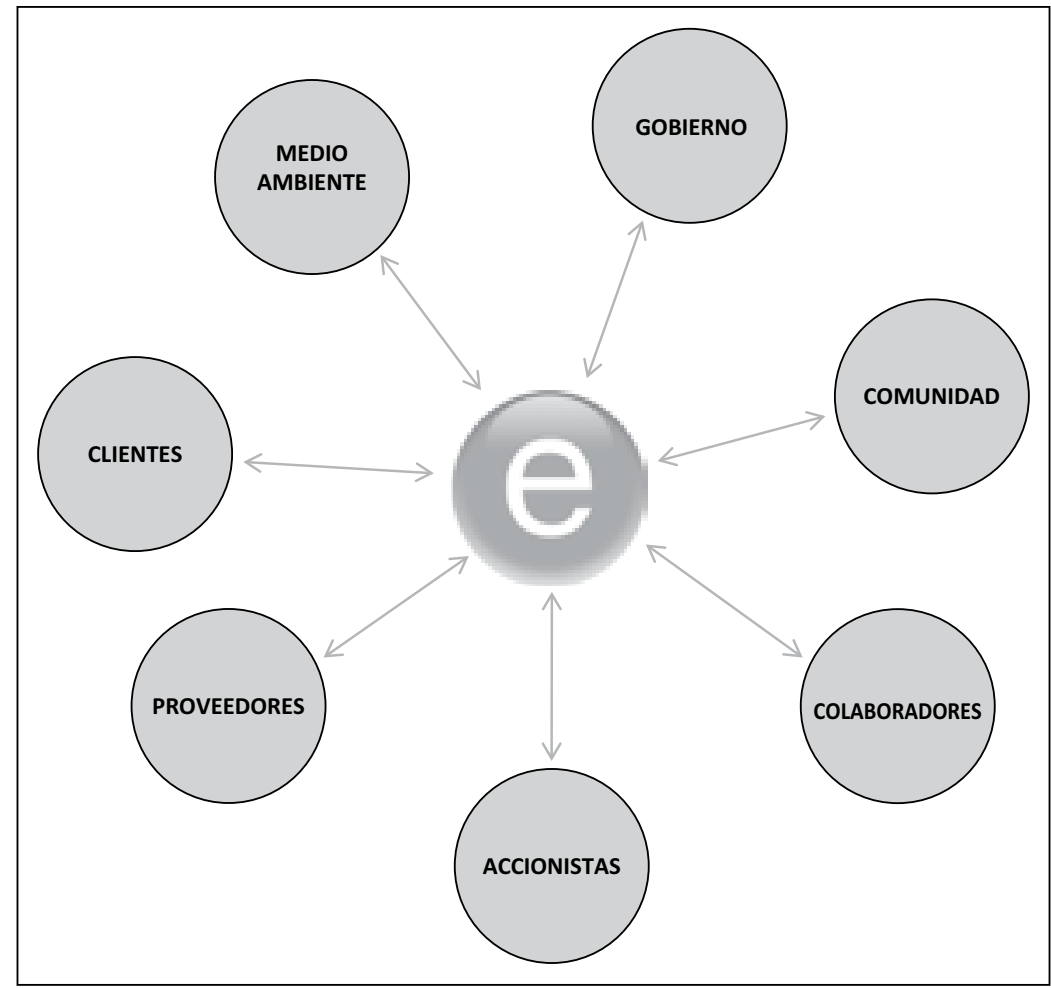

Fuente: ISO 
Figura 3. Dimensiones de los impactos

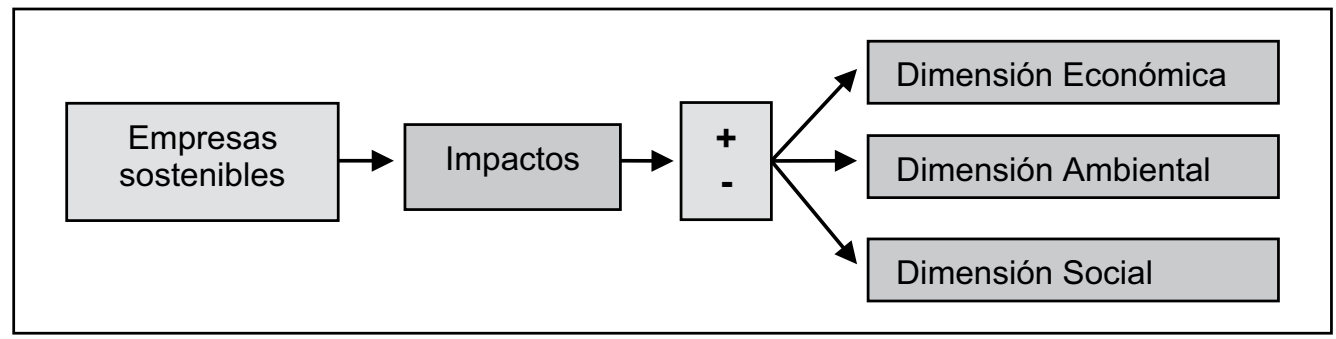

Fuente: Elaboración propia.

la económica. La dimensión ambiental y social se analiza por separado porque tienen enfoques diferentes, el primero analiza los impactos en el ambiente propiamente dicho con sus metodologías e instrumentos; siendo ya tratada por ISO 14000.

La dimensión social debe analizar los impactos (modificación de: costumbres, cultura, hábitos de vida, intereses, coordinaciones, interacciones, etc.) que genera la empresa en los distintos grupos de interés relacionados a la comunidad; los que influyen en su sostenibilidad. Bajo esa perspectiva no se presentaría duplicidad de enfoques entre la dimensión ambiental y social como ocurre en muchos instrumentos.

La primera observación al enfoque que propone ISO se presenta en el "medio ambiente", como tal, no es un grupo de interés propiamente dicho; las actividades de la empresa y su relación con sus grupos siempre se desarrollan en un "medio ambiente" y claro bajo una responsabilidad de generar impactos sobre ella. El cumplimiento normativo sobre aspectos legales relacionados al ambiente, son parte de los intereses del gobierno, cuyas políticas establecen instrumentos como la evaluación de impacto ambiental y el programa de adecuación ambiental.

La responsabilidad social debe tratar temas relacionados solo a los grupos de interés; es decir, para los colaboradores y accionistas debe existir un trato cordial respetando los derechos de todos, actuando en lo correcto, con una transparencia en la comunicación, incentivando la solidaridad e integración.

A los clientes, proveedores y gobierno se debe dar énfasis en el "trato justo", transparente y equitativo, respetando los intereses de cada uno de ellos.

Figura 4. Nuevo esquema de modelo de RS

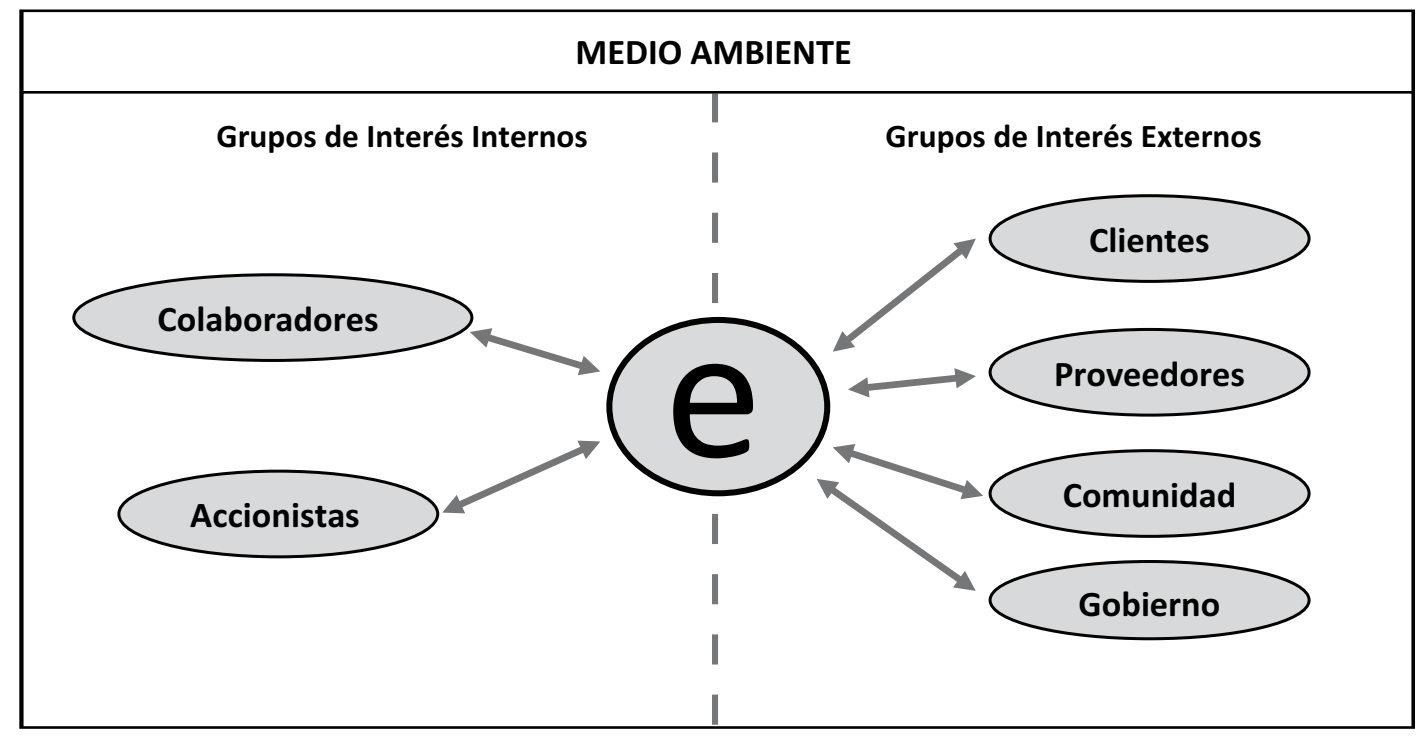

Fuente: Elaboración propia. 
La comunidad es un grupo de suma importancia ya que ella otorga la "licencia social" es decir, las opiniones, percepciones y aceptación de la comunidad es a favor del desempeño de la empresa, no solo apoyándola sino integrándola en sus actividades colectivas. En la figura 4 se integra los conceptos anteriores, generando el enfoque modificado de la responsabilidad social:

Entonces, se puede resumir que la responsabilidad social implica una actuación correcta de la organización con todos sus grupos de interés $\mathrm{y}$ se desenvuelven en un medio ambiente con responsabilidad.

\section{CONCLUSIONES}

1. Para analizar en forma integrar los impactos de las empresas es necesario agruparlas en tres dimensiones: económica, ambiental y social.

2. Los grupos de interes relacionados a las empresas se enmarcan en las dimensiones economica y social.

3. El medio ambiente por si, no cumple las caracteristicas de grupo de interes.

4. Es necesario modificar el enfoque conceptual de ISO 26000 sobre responsabilidad social por aquel que analice a los grupos de interés y al medio ambiente en forma independiente.

\section{RECOMENDACIONES}

1. Incluir la cultura de aplicar el enfoque de responsabilidad social en todas las organizaciones indistintamente de su clasificación para lograr su sostenibilidad a largo plazo.

\section{REFERENCIAS BIBLIOGRÁFICAS}

[1] Andia, W. (2010). Gerencia de Poryectos Sociales y de Inversion Social. Lima, Perú: El saber.

[2] Ardagoña, A. y Isea, R. (2011). ISO 26000, Una Guía para la Responsabilidad Social de las Organizaciones. Cuadernos de la Cátedra "La Caixa" de Responsabilidad Social de la Empresa y Gobierno Corporativo, 11, p. 8-10. Recuperado de http://comunicarseweb. com.ar/download.php?tipo=acrobat\&view $=1 \& d$ ato $=1308234290 \_c a t e d r a l a c a i x a \_v o l 11$ Final_ tcm5-66526.pdf

[3] Guía ISO 26000 Responsabilidad Social (18 de Julio de 2014) Global STD, p. 1-2. Recuperado de http://www.globalstd.com/networks/blog/ guia-iso-26000-responsabilidad-social

[4] Olcese, A., Rodríguez, M. y Alfaro, J. (2008). Manual de la Empresa Responsable y Sostenible. Madrid, España: McGraw-Hill. 